\title{
Ultraviolet Behavior of Non-Abelian Gauge Theories*
}

\author{
David J. Gross † and Frank Wilczek \\ Joseph Henry Laboratories, Princeton University, Princeton, New Jersey 08540
}

(Received 27 April 1973)

\begin{abstract}
It is shown that a wide class of non-Abelian gauge theories have, up to calculable logarithmic corrections, free-field-theory asymptotic behavior. It is suggested that Bjorken scaling may be obtained from strong-interaction dynamics based on non-Abelian gauge symmetry.
\end{abstract}

Non-Abelian gauge theories have received much attention recently as a means of constructing unified and renormalizable theories of the weak and electromagnetic interactions.' In this note we report on an investigation of the ultraviolet (UV) asymptotic behavior of such theories. We have found that they possess the remarkable feature, perhaps unique among renormalizable theories, of asymptotically approaching free-field theory. Such asymptotically free theories will exhibit, for matrix elements of currents between on-mass-shell states, Bjorken scaling. We therefore suggest that one should look to a non-Abelian gauge theory of the strong interactions to provide the explanation for Bjorken scaling, which has so far eluded field-theoretic understanding.

The UV behavior of renormalizable field theories can be discussed using the renormalization-group equations, ${ }^{2,3}$ which for a theory involving one field (say $g \varphi^{4}$ ) are

$$
[m \partial / \partial m+\beta(g) \partial / \partial g-n \gamma(g)] \mathbf{\Gamma}_{\text {asy }}{ }^{(n)}\left(g ; P_{1}, \ldots, P_{n}\right)=0 \text {. }
$$

$\Gamma_{\text {asy }}{ }^{(n)}$ is the asymptotic part of the one-particle-irreducible renormalized $n$-particle Green's function, $\beta(g)$ and $\gamma(g)$ are finite functions of the renormalized coupling constant $g$, and $m$ is either the renormalized mass or, in the case of massless particles, the Euclidean momentum at which the theory is renormalized. ${ }^{4}$ If we set $P_{i}=\lambda q_{i}{ }^{0}$, where $q_{i}{ }^{0}$ are (nonexceptional) Euclidean momenta, then (1) determines the $\lambda$ dependence of $\Gamma^{(n)}$ :

$$
\Gamma^{(n)}\left(g ; P_{i}\right)=\lambda^{D} \Gamma^{(n)}\left(\bar{g}(g, t) ; q_{i}\right) \exp \left[-n \int_{0}^{t} \gamma\left(\bar{g}\left(g, t^{\prime}\right)\right) d t^{\prime}\right],
$$

where $t=\ln \lambda, D$ is the dimension (in mass units) of $\Gamma^{(n)}$, and $\bar{g}$, the invariant coupling constant, is the solution of

$$
d \bar{g} / d t=\beta(\bar{g}), \quad \bar{g}(g, 0)=g .
$$

The UV behavior of $\Gamma^{(n)}(\lambda \rightarrow+\infty)$ is determined by the large-t behavior of $\bar{g}$ which in turn is controlled by the zeros of $\beta$ : $\beta\left(g_{f}\right)=0$. These fixed points of the renormalization-group equations are said to be UV stable [infrared (IR) stable] if $\bar{g} \rightarrow g_{f}$ as $t \rightarrow+\infty(-\infty)$ for $\bar{g}(0)$ near $g_{f}$. If the physical coupling constant is in the domain of attraction of a UV-stable fixed point, then

$$
\Gamma^{(n)}\left(g ; P_{i}\right) \underset{\lambda \rightarrow \infty}{\approx} \lambda^{D-n \gamma\left(g_{f}\right)} \Gamma^{(n)}\left(g_{f} ; q_{i}\right) \exp \left\{-n \int_{0}^{\infty}\left[\gamma(\bar{g}(g, t))-\gamma\left(g_{f}\right)\right] d t\right\},
$$

so that $\gamma\left(g_{f}\right)$ is the anomalous dimension of the field. As Wilson has stressed, the UV behavior is determined by the theory at the fixed point ( $g$ $\left.=g_{f}\right) .^{5}$

In general, the dimensions of operators at a fixed point are not canonical, i.e., $\gamma\left(g_{f}\right) \neq 0$. If we wish to explain Bjorken scaling, we must as sume the existence of a tower of operators with canonical dimensions. Recently, it has been argued for all but gauge theories, that this can only occur if the fixed point is at the origin, $g_{f}=0$, so that the theory is asymptotically free. ${ }^{6,7}$ In that case the anomalous dimensions of all operators vanish, one obtains naive scaling up to finite and calculable powers of $\ln \lambda$, and the structure of operator products at short distances is that of free-field theory. ${ }^{7}$ Therefore, the existence of such a fixed point, for a theory of the strong interactions, might explain Bjorken scaling and the success of naive light-cone or parton-model relations. Unfortunately, it appears that the fixed point at the origin, which is common to all theories, is not UV stable. ${ }^{8,9}$ The only exception would seem to be non-Abelian gauge theories, which hitherto have not been explored in this re- 
gard.

Let us consider a Yang-Mills theory given by the Lagrangian

$$
\begin{aligned}
& \mathscr{L}=-\frac{1}{4} F_{\mu \nu}{ }^{a} F_{a}{ }^{\mu \nu}, \\
& F_{\mu \nu}{ }^{a}=\partial_{\mu} A_{\nu}{ }^{a}-\partial_{\nu} A_{\mu}{ }^{a}-g C_{a b c} A_{\mu}{ }^{b} A_{\nu}{ }^{c},
\end{aligned}
$$

where the $C_{a b c}$ are the structure constants of some (semisimple) Lie group $G$. Since the theory is massless, the renormalization is performed at an (arbitrary) Euclidean point. For example, the wave-function renormalization constant $Z_{3}(g$, $\Lambda / m)$ will be defined in terms of the unrenormalized vector-meson propagator $D_{\mu \nu}{ }^{a b}$ (in the Landau gauge),

$$
\left.D_{\mu \nu}{ }^{a b}(P)\right|_{F^{2}=-m^{2}}=\left(g_{\mu \nu}+\frac{P_{\mu} P_{\nu}}{m^{2}}\right) \frac{i Z_{3}}{m^{2}} \delta_{a b} .
$$

(For a thorough discussion of the renormalization see the work of Lee and Zinn-Justin. ${ }^{10}$ ) The renormalization-group equations for this theory are easily derived. ${ }^{11}$ In the Landau gauge they are identical with (1). In order to investigate the stability of the origin, it is sufficient to calculate $\beta$ to lowest order in perturbation theory. To this order we have

$$
\beta(g)=\left.\frac{\partial g}{\partial \ln m}\right|_{\Lambda_{1} g_{0}}=-g \frac{\partial}{\partial \ln \Lambda}\left(\frac{Z_{3}{ }^{3 / 2}}{Z_{1}}\right),
$$

where $\Lambda$ is a UV cutoff, and $Z_{1}$ the charge-renormalization constant. In Abelian gauge theories $Z_{3}=Z_{1}=1-g^{2} C \ln \Lambda(C>0)$, as a consequence of gauge invariance and the Källén-Lehman representation, and thus $\beta(g) \cong g^{3}$ which leads to IR stability at $g=0$. Non-Abelian theories have no such requirement; $Z_{3}$ and $Z_{1}$ are gauge dependent and can be greater than 1 . Thus $\beta(g)$, which must be gauge independent in lowest order, could have any sign at $g=0$. We have calculated $Z_{1}$ and $Z_{3}$ for the above Lagrangian, and we find that ${ }^{12}$

$$
\beta_{V}=-\left(g^{3} / 16 \pi^{2}\right) \frac{11}{3} C_{2}(G)+O\left(g^{5}\right),
$$

where $C_{2}(G)$ is the quadratic Casimir operator of the adjoint representation of the group $G: \sum_{b, c} c_{a b c}$ $\times c_{d b c}=C_{2}(G) \delta_{a d}\left[\right.$ e.g., $\left.C_{2}(\mathrm{SU}(N))=N\right]$. The solution of (3) is then $\bar{g}^{2}(t)=g^{2} /\left(1-2 \beta_{V} g^{-1} t\right)$, and $\bar{g}$ $\rightarrow 0$ as $t \rightarrow \infty$ as long as the physical coupling constant $g$ is in the domain of attraction of the origin. ${ }^{13}$

We have thus established that for all non-Abelian gauge theories based on semisimple Lie groups the origin is UV stable. It is easy to incorporate fermions into such a theory without destroying the UV stability. The fermion interac- tion is given by $L_{F}=\bar{\psi}\left(i \gamma \cdot \delta-g \gamma \cdot B^{a} M^{a}\right) \psi+$ mass terms, where $M^{a}$ are the matrices of some representation $R$ of the gauge group $G$. The only effect of the fermions is to change the value of $\beta(g)$ by the amount ${ }^{11}$

$$
\beta_{\boldsymbol{F}}(R)=\left(g^{3} / \mathbf{1 6} \pi^{2}\right) \frac{4}{3} T(R),
$$

where $\operatorname{Tr}\left(M^{a} M^{b}\right)=T(R) \delta_{a b}, T(R)=C_{2}(R) d(R) / r$, $d(R)$ is the dimension of the representation $K$, and $r$ is the order of the group, i.e., the number of generators, and $C_{2}(R)$ is the quadratic Casimir operator of the representation. Although the fermions tend to destabilize the origin, there is room to spare. For example, in the case of $\mathrm{SU}(3): \beta_{v}=-11$, whereas $\beta_{F}(\underline{3})=\frac{2}{3}, \beta_{F}(\underline{8})=4$, etc., so that one could accomodate as many as sixteen triplets. One can therefore construct many asymptotically free theories with fermions. The vector mesons, however, will remain massless until the gauge symmetry is spontaneously broken. One might hope that this would be a consequence of the dynamics, ${ }^{14}$ but at the present the only known way of achieving this is to introduce scalar Higgs mesons, whose nonvanishing vacuum expectation values break the symmetry.

The introduction of scalar mesons has a very destabilizing effect on the UV stability of the origin. Their contribution to $\beta(g)$ is small; a scalar meson transforming under a complex (real) representation $R$ of the gauge group adds to $\beta$ a term equal to $\frac{1}{4}\left(\frac{1}{8}\right)$ of Eq. (9). The problem with scalar mesons is that they necessarily have their own quartic couplings, and one must deal with a new coupling constant. Consider the Lagrangian for the coupling of scalars belonging to a representation $R$ of $G$ :

$$
\mathscr{L}=\frac{1}{2}\left[\left(\partial_{\mu}-i g B_{\mu}{ }^{a} M^{a}\right) \vec{\varphi}\right]^{2}-\lambda(\vec{\varphi} \cdot \vec{\varphi})^{2}+V(\vec{\varphi}) .
$$

where $V(\varphi)$ contains cubic, quadratic, and linear terms in $\varphi$ (which have no effect on the UV behavior of the theory) plus, perhaps, additional quartic terms invariant under $G$. The renormalization-group equations have an additional term, $\beta_{\lambda}(g, \lambda) \partial / \partial \lambda$, and one must investigate the UV stability of the origin $(g=\lambda=0)$ with respect to both $g$ and $\lambda$ [if there are other quartic invariants in $V(\varphi)$ there will be additional coupling constants to consider]. The structure of the renormalization-group equation for $g$ is unchanged to lowest order, whereas for the coupling constant $I \equiv \lambda / g^{2}$ we have ${ }^{11}$

$$
d \bar{\Gamma}\left(\Gamma, t, g^{2}\right) / d t=\bar{g}^{2}\left[A \bar{\Gamma}^{2}+B \bar{\Gamma}+C\right]
$$

(where we have neglected terms of order $g^{4}, g^{4} \Gamma$, 
$g^{4} \Gamma^{2}$, and $\left.g^{4} \Gamma^{3}\right)$. In the absence of vector mesons $(g=0)$ this equation is UV unstable at $\lambda=0$, since $A$ is strictly positive and $\lambda$ must be positive. ${ }^{15}$ The vector mesons contribute to $B$ and $C$ and tend to stabilize the origin. If the right-hand side of (11) has positive zeros $\left(C>0, B<0\right.$, and $B^{2}$ $>4 A C$ ), then for $\Gamma$ less than the larger zero of (11) we will have that $\lambda \rightarrow+0$ as $t \rightarrow \infty$. We have investigated the structure of these equations for a large class of gauge theories and representations of the scalar mesons. We have found many examples of theories which contain scalar mesons and are UV stable. ${ }^{11}$ These include (a) $\mathrm{SU}(N)$ if the scalar mesons belong to the adjoint representation for $N \geqslant 6$; (b) $\mathrm{SU}(N) \otimes \mathrm{SU}(N)$ if the scalars belong to the $(N, \bar{N})$ representation for $N \geqslant 5$; (c) $\mathrm{SU}(N)$ with the scalars transforming as a symmetric tensor for $N \geqslant 9$; and many others. In all of these models it is necessary for the theory to contain a large number of fermions in order to make $\beta_{g}$ small; otherwise $\bar{g}$ approaches zero too rapidly for the vector mesons to stabilize the scalar couplings.

Unfortunately, in none of these models can the gauge symmetry be totally broken by the Higgs mechanism. The requirement that the interactions of the scalar mesons be renormalizable so severely constrains the form of Lagrangian that the ground state invariably is invariant under some non-Abelian subgroup of the gauge group. If one tries to overcome this by larger representations for the scalar mesons, UV instability inevitably occurs.

It thus appears to be very difficult to retain UV stability and break the gauge symmetry by explicitly introducing Higgs mesons. Since the Higgs mesons are so restrictive, we would prefer to believe that spontaneous symmetry breaking would arise dynamically..$^{14}$ This is suggested by the IR instability of the theories, which assures us that perturbation theory is not trustworthy with respect to the stability of the symmetric theory nor to its particle content.

With this hope in mind one can construct many interesting models of the strong interactions. One particularly appealing model is based on three triplets ${ }^{16}$ of fermions, with Gell-Mann's $\mathrm{SU}(3) \otimes \mathrm{SU}(3)$ as a global symmetry and an $\mathrm{SU}(3)$ "color" gauge group to provide the strong interactions. That is, the generators of the stronginteraction gauge group commute with ordinary $\mathrm{SU}(3) \otimes \mathrm{SU}(3)$ currents and mix quarks with the same isospin and hypercharge but different "color." In such a model the vector mesons are neutral, and the structure of the operator product expansion of electromagnetic or weak currents is (assuming that the strong coupling constant is in the domain of attraction of the origin!) essentially that of the free quark model (up to calculable logarithmic corrections). ${ }^{11}$

Finally, we note that theories of the weak and electromagnetic interactions, built on semisimple Lie groups,${ }^{17}$ will be asymptotically free if we again ignore the complications due to the Higgs particles. This suggests that the program of Baker, Johnson, Willey, and Adler ${ }^{18}$ to calculate the fine-structure constant as the value of the UV-stable fixed point in quantum electrody namics might fail for such theories.

*Research supported by the U.S. Air Force Office of Scientific Research under Contract No. F-44620-71-C0180 .

$\dagger$ Alfred P. Sloan Foundation Research Fellow.

${ }^{1}$ S. Weinberg, Phys. Rev. Lett. 19, 1264 (1967). For an extensive review as well as a list of references, see B. W. Lee, in Proceedings of the Sixteenth International Conference on High Energy Physics, National Accelerator Laboratory, Batavia, Illinois, 1972 (to be published).

${ }^{2}$ M. Gell-Mann and F. E. Low, Phys. Rev. 95, 1300 (1954) .

${ }^{3}$ C。G. Callan, Phys. Rev. D 2 , 1541 (1970); K. Symanzik, Commun. Math. Phys. 18, 227 (1970).

${ }^{4}$ The basic assumption underlying the derivation and utilization of the renormalization group equations is that the large Euclidean momentum behavior of the theory is the same as the sum, to all orders, of the leading powers in perturbation theory.

${ }^{5} \mathrm{~K}$. Wilson, Phys. Rev. D $\underline{3}, 1818$ (1971).

${ }^{6} \mathrm{G}$. Parisi, to be published。

${ }^{7}$ C. G. Callan and D. J. Gross, to be published.

${ }^{8} \mathrm{~A}$. Zee, to be published.

${ }^{9} \mathrm{~S}$. Coleman and D. J. Gross, to be published.

${ }^{10}$ B. W. Lee and J. Zinn-Justin, Phys. Rev. D $\underline{5}, 3121$ (1972).

${ }^{11}$ Full details will be given in a forthcoming publication: D. J. Gross and F. Wilczek, to be published.

${ }^{12}$ After completion of this calculation we were informed of an independent calculation of $\beta$ for gauge theories coupled to fermions by H. D. Politzer [private communication, and following Letter, Phys. Rev. Lett. 30, 1346 (1973)]。

${ }^{13} \mathrm{~K}$. Wilson has suggested that the coupling constants of the strong interactions are determined to be IRstable fixed points. For nongauge theories the IR stability of the origin in four-dimensional field theories implies that theories so constructed are trivial, at least in a domain about the origin. Our results suggest that non-Abelian gauge theories might possess IR-stable fixed points at nonvanishing values of the coupling constants. 


\footnotetext{
${ }^{14}$ Y. Nambu and G. Jona-Lasino, Phys. Rev. 122, 345 (1961); S. Coleman and E. Weinberg, Phys. Rev. D 7 , 1888 (1973).

${ }^{15} \mathrm{~K}$. Symanzik (to be published) has recently suggested that one consider a $\lambda \varphi^{4}$ theory with a negative $\lambda$ to achieve UV stability at $\lambda=0$ 。 However, one can show, using the renormalization-g roup equations, that in such theory the ground-state energy is unbounded from below (S. Coleman, private communication).
}

${ }^{16} \mathrm{~W}$ 。A. Bardeen, H. Fritzsch, and M。Gell-Mann, CERN Report No. CERN-TH-1538, 1972 (to be published)。

${ }^{17} \mathrm{H}$. Georgi and S. L. Glashow, Phys. Rev. Lett. 28, 1494 (1972); S。Weinberg, Phys. Rev. D 5 , 1962 (1972). ${ }^{18}$ For a review of this program, see $S$ 。 Proceedings of the Sixteenth International Conference on High Energy Physics, National Accelerator Laboratory, Batavia, Illinois, 1972 (to be published).

\title{
Reliable Perturbative Results for Strong Interactions?*
}

\author{
H. David Politzer \\ Jefferson Physical Laboratories, Harvard University, Cambridge, Massachusetts 02138 \\ (Received 3 May 1973)
}

An explicit calculation shows perturbation theory to be arbitrarily good for the deep Euclidean Green's functions of any Yang-Mills theory and of many Yang-Mills theories with fermions. Under the hypothesis that spontaneous symmetry breakdown is of dynamical origin, these symmetric Green's functions are the asymptotic forms of the physically significant spontaneously broken solution, whose coupling could be strong.

Renormalization-group techniques hold great promise for studying short-distance and strongcoupling problems in field theory. ${ }^{1,2}$ Symanzik $^{2}$ has emphasized the role that perturbation theory might play in approximating the otherwise unknown functions that occur in these discussions. But specific models in four dimensions that had been investigated yielded (in this context) disappointing results. ${ }^{3}$ This note reports an intriguing contrary finding for any generalized Yang-Mills theory and theories including a wide class of fermion representations. For these one-coupling-constant theories (or generalizations involving product groups) the coefficient function in the Callan-Symanzik equations commonly called $\beta(g)$ is negative near $g=0$.

The constrast with quantum electrodynamics (QED) might be illuminating. Renormalization . of QED must be carried out at off-mass-shell points because of infrared divergences. For small $e^{2}$, we expect perturbation theory to be good in some neighborhood of the normalization point. But what about the inevitable logarithms of momenta that grow as we approach the mass shell or as some momenta go to infinity? In QED, the mass-shell divergences do not occur in observable predictions, when we take due account of the experimental situation. The renormalization-group technique ${ }^{4}$ provides a somewhat opaque analysis of this situation. Loosely speaking, ${ }^{5}$ the effective coupling of soft photons goes to zero, compensating for the fact that there are more and more of them. But the large$p^{2}$ divergence represents a real breakdown of perturbation theory. It is commonly said that for momenta such that $e^{2} \ln \left(p^{2} / m^{2}\right) \sim 1$, higher orders become comparable, and hence a calculation to any finite order is meaningless in this domain. The renormalization group technique shows that the effective coupling grows with momenta.

The behavior in the two momentum regimes is reversed in a Yang-Mills theory. The effective coupling goes to zero for large momenta, but as $p^{2}$ 's approach zero, higher-order corrections become comparable. Thus perturbation theory tells nothing about the mass-shell structure of the symmetric theory. Even for arbitrarily small $g^{2}$, there is no sense in which the interacting theory is a small perturbation on a free multiplet of massless vector mesons. The truly catastrophic infrared problem makes a symmetric particle interpretation impossible. Thus, though one can well approximate asymptotic Green's functions, to what particle states do they refer?

Consider theories defined by the Lagrangian

$$
\mathfrak{L}=-\frac{1}{4} F_{\mu \nu}^{a} F^{a \mu \nu}+i \bar{\psi}_{i} \gamma \cdot D_{i j} \psi_{j},
$$

where

$$
F_{\mu \nu}{ }^{a}=\partial_{\mu} A_{\nu}{ }^{a}-\partial_{\nu} A_{\mu}{ }^{a}+g f^{a b c} A_{\mu}{ }^{b} A_{\nu}{ }^{c},
$$

